\title{
Materials of the round table "Analytical methods and computer experiment in soft matter theory"
}

\author{
Collected and edited by \\ A. Trokhymchuk ${ }^{1}$, D.Henderson ${ }^{2}$, L.Lee ${ }^{3}$ \\ 1 Institute for Condensed Matter Physics, National Academy of Science of \\ the Ukraine, Lviv, UA 79011, Ukraine \\ 2 Department of Chemistry and Biochemistry, Brigham Young University, \\ Provo, UT 84602, USA \\ 3 School of Chemical Engineering and Material Science, University of \\ Oklahoma, Norman, OK 73019, USA
}

\begin{abstract}
The paper contains materials of the discussion regarding the development, the problems of today and the prospects of analytical methods and computer simulations in soft matter theory which took place during the Workshop on modern problems of soft matter theory (August 27-31, 2000, Lviv, Ukraine).
\end{abstract}

Key words: analytical theory, computer simulations

PACS: 07.05.T

\section{In lieu of introduction}

The last decade of the 20th century gave mixed prospects for the future developments of theoretical [i.e., analytical] approaches in the soft matter theory that traditionally includes thermodynamic perturbation theory, integral equation theory and density functional theory. Specifically, it may be not very promising to apply theoretical approaches to sophisticated models in the soft matter theory that have become very popular in different applications. Many basic and analytically solvable models have been already solved within some physically reasonable approximations.

Rapid development of computer technology creates the necessary conditions for the expansion of computer experiment methods that can be considered as a third way to investigate condensed matter in between theory and experiment: Two basic methods of computer experiment have been developed: Monte Carlo (MC) and Molecular Dynamics (MD). In both methods, a certain number of particles is distributed in a (usually cubic) box. The interactions are described by pair potentials 
and it is generally assumed that the total potential can be developed as a sum of these pair potentials.

In $M C$ simulations, a large number of configurations are generated. Starting from a given configuration, a trial move of a randomly chosen particle to a new position is made. The energy change, $\Delta E$, connected with this move is calculated, and if the energy decreases, the move is accepted. If $\Delta E$ is positive, the Boltzmann factor $\exp \left(-\Delta E / k_{B} T\right)$ is calculated and compared with a randomly chosen number between 0 and 1 . The move is accepted if the Boltzmann factor is larger than this number and rejected if it is smaller. This way, millions of configurations, weighted by the Boltzmann factor, are produced and thermodynamics and structural properties of the studied system can be deduced by averaging procedures. In MD simulations, the classical equations of motion are integrated numerically for the particles in the box. The resulting knowledge of position and velocity is determined as a function of time for each of the particles and means a complete classical description of the system. Therefore, the thermodynamic, structural as well as dynamic properties of the liquid concerned can be calculated using statistical mechanics. A comprehensive description of both methods has been given by Allen and Tildesley [1] and Frenkel and Smit [2].

With the disposal of such powerful tools, one can comment that he has no need for analytical theory, saying that simulations can provide the numbers that are needed. However, the purpose of theory was not to provide numbers but to provide understanding. For example, Copernicus, and more especially Kepler, inferred from astronomical data, mostly due to Brahe, the details of the planetary orbits. However, this provided no understanding. Surely, understanding and establishing interconnections is the goal of science. It was Newton's analytical work which established that Kepler's laws were a consequence of gravitational attraction with a simple inverse square dependence together with the conservation of angular momentum. It is unlikely that such far reaching concepts could have been achieved without analytical work. Perhaps even more importantly, the fact that the same laws governed astronomical motions over much larger distances [the structure of nebulae is but one example] would never have been discovered without the developments based on Newton's analytical theories. Would quantum mechanics have been discovered without Planck and Bohr feeding their ideas into analytical formulae? Would simulations have aided Maxwell or Einstein?

The future developments of the soft matter theory will determine the role of analytical theories and computer experiment. However, scientists are trying to forecast the future. The best way to do this is to have an understanding of the past and a critical view what is going in the present. From our experience, we can say that such kind of questions recently arise very often within scientists in their private communications, discussions during group meetings and large scale conferences. Nevertheless, no materials regarding those discussions are published so far. Partial insight into this topic has been made during the two-hour round table conversation organized within the Workshop on Modern Problems of Soft Matter Theory [August 27-31, 2000, Lviv, Ukraine]. We avoid to make some final conclusions following that 
discussion. This is not because of they were not formulated, but because we are not sure that one could do this in general. Thus, the goal of this publication is to present for a larger number of scientists the view of the participants of the round table and, perhaps, to initiate the future publications on this topic.

\section{The role of analytical methods and computer experiment in the soft matter theory}

Karl Heinzinger: Computer simulations, being neither experiment nor theory, can take over the task of both. In the case of simple liquids, say liquid noble gases, where the interactions between the particles are well known and where many-body non-additive interactions do not play an important role, the results derived from the simulations deserve a high degree of reliability. They can be used as a standard either for or against analytical theories. In the case of complicated systems, say interfaces, that cannot yet be treated analytically, and computer simulations can play the role of the theory. They can predict properties of liquids which cannot [or not directly] be measured and they can explain macroscopically measured properties on a molecular level.

Myroslav Holovko: Analytical methods and computer experiments are complementary to one other. This is similar to the relationships that we have between theory and real experiments. The information obtained from computer simulations should be reproduced by an analytical theory. This gives a test for analytical methods but is not limited only by this. In the process of such a comparison we learn more and understand more about the properties and phenomena in model systems.

Marco Zoppi: In my opinion the two techniques are complementary. On the one hand, computer experiments are a way to solve a well defined model up to a preselected precision level. For example, you can decide to solve the problem of a pairwiseadditive Lennard-Jones liquid system, which however does not exist in nature, but you can do it almost exactly using simulation. On the other hand, to solve the same model system, analytically, is not possible, unless you resort to approximations, which in turn, may or may not be fully controlled. A comparison between the two methods, and the results, can shed some light on the effects and applicability range of the approximations. This, in turn, would improve the effectiveness of the analytical method, giving a rather precise consciousness of its applicability limits.

Fumio Hirata: I wish to express my opinion on a general question which has often been raised concerning to the importance of the integral equation methods in liquid state theory. Why does one use the integral equation method to solve the problems when the molecular simulation can replace it in many cases? We can answer the question in two ways: one technical and the other more fundamental. Due to the level of complication which characterizes the problems in chemistry, solutions to statistical-mechanical equations are numerical in most of the cases. In that sense, statistical-mechanical equations apparently are not any better than molecular simulations. However, there are problems in which the molecular simulation fails to give an answer to a question within a reasonable amount of computational resources cur- 
rently available. A typical example of such questions is the protein folding based on the first principles. For such cases, the difference between the statistical-mechanical theory and the molecular simulations is not just technical but essential.

Dor Ben-Amotz: Obviously numerical methods are increasing in importance with the evolving computer technology, as numerical methods may be used to model the systems that are far too complex to treat analytically (at least at the same level of detail). However, it is analytical theory that provides the language we use to formulate the key issues and questions which are most interesting and important, including those whose answers may require numerical or laboratory experiments. Analytical theory also represents the sacred heart of all scientific endeavour, as it is this branch of research which expresses our deep seated belief in the mystery of all mysteries - that we live in a world which may yield itself to our understanding if we think hard and have sufficient faith.

Lesser Blum: I believe that as scientists we have a common goal, which is to understand nature. In our case, statistical mechanics tries to establish the connection between molecular forces (which cannot be directly measured) and the behaviour of macroscopic systems. The name of the game is to formulate simple enough theories which from a given Hamiltonian model can predict the behaviour of systems of variable complexity. The link between pure theory and pure experiment is provided by simulation.

Marco Zoppi: Here I have a similar opinion that the problem is ill posed. Analytical theories and computer experiments can be dealing with exactly the same model system, which, however, can be totally unrealistic. On the other hand, it is not always true that a computer experiment "probes" the reality. Most of the times it gives answers, or numbers, which depend critically on he way we have modelled the reality. Of course, IF we could have an infinitely precise potential model (including all the many-body contributions) IF we could demonstrate that a limited simulation sample is a good representative of a real physical system, IF the simulation algorithm worked perfectly well, THEN the simulation results should mimic exactly the reality. But, how sure are we about all those IFs? Of course, if we enclose the concept of an error bar, most of the problems can be released, or decreased, but HOW do we get rid of the unavoidable systematic errors that are always present in the simulations? [and also in the experiments, of course.]

Yura Kalyuzhnyi: In my opinion, our discussion has focused only on the role of the theory from the short-term viewpoint. However it is important to stress the role of the theory on a long-term scale. In this sense, the theory is very important, since it gives us the definitions [or terminology] and the language, which is used not only in experimental studies or in engineering but in the whole society. For example one can hardly imagine any field of engineering without using the definition of the energy. However, a couple of centuries ago this terminology did not exist and appeared as a result of the studies that were mostly theoretical.

Fumio Hirata: There is an even more fundamental reason to develop the statistical mechanical theories. Let us explain this point by taking just one example from the solution chemistry. One of the most important findings in the solution chemistry 
is the so-called "square root law" which appears in many of equilibrium and nonequilibrium properties in electrolyte solutions in their concentration dependence: the asymptotic concentration dependence in the $\lim C \rightarrow 0$ is proportional to $C^{1 / 2}$, not to $C$. As has been clarified by the statistical mechanics of solutions, the limiting behaviour is intimately related to the multi-body screening of the Coulomb interaction among charged species. In the typical electrolyte solutions, the Coulomb interaction between a pair of ions asymptotically behaves as $\mathrm{e}^{-\kappa r} / \epsilon r$, that is, the Coulomb interaction is screened by an exponential factor $\mathrm{e}^{-\kappa r}$, where $\kappa$ is th Debye screening factor. The square root dependence of the screening constant upon concentrations appearing in this equation is the physical origin of the limiting law. The exponential screening behaviour is obtained mathematically from the integral equation theory as a renormalization of the coulomb interactions. That is, the physically realized phenomenon of screening has a clear "mathematical" interpretation based on the statistical mechanical theory. Although the molecular simulation may reproduce the exponential behaviour of screening in any precision, it will never answer the question why it should be exponential, why it should produce the special concentration dependence for many observable quantities.

Myroslav Holovko: However, the role of computer simulation continues to increase. We can predict that this will continue in near future. Advanced simulation methods lead to new insights and simulate new suggestions for improvements in analytical studies. As examples of such new developments, we can consider the multidensity integral equation approach in the theory of associating and polymeric fluids, the replica Ornstein-Zernike integral equations for the liquids in porous media, the application of the integral equation approach to the description of electronic density of states of fluids and so on.

\section{Prospects for Soft Matter Theory}

Marco Zoppi: Personally I am more in favour of the applications of the existing methods to modern objects. Probably because I am not a professional in simulation. When you have some new experimental results, the first problem is to validate them somehow. In this case a simulation can be very helpful. From this stage on, to find the physical insight is not a trivial process and you may need some theoretical approach. At any rate, developing new methods is always a knowledge increasing process and sooner or later it will give its fruit.

Dor Ben-Amotz: In more practical terms, the importance of numerical experiments in testing analytical theory is well appreciated. There is some danger, however, that analytical theory and simulation can lead each other astray as they dance happily together off the edge of anything resembling the real world. A good example is our fondness for two-body additivity in modeling fluids and complex materials. Both analytical theory and numerical simulations are quite happy to follow this path, as they pat each other on the back congratulating each others success, in spite of the fact that interactions between the molecules of water in this beer bottle bear no resemblance to such two-body additive potentials. 
Lesser Blum: In my perception, all three methods, i.e. theory, simulations, experiment, are important. It is like the old story of which is the most important part of the body: the head, the heart, or the stomach: We need all three. A case in point is the biology study at the molecular level in general, and of biochannels in particular. These are extremely important, complex and delicately precise structures which even with the most powerful computers cannot be simulated in any even remotely close to physiological conditions, for sufficiently long times. And theoretical approaches are still too simplistic to account for the delicate mechanism of gating.

Myroslav Holovko: Analytical approaches can be developed before the computer simulations. As an example of such a situation, we have the theory of self-assembling systems, where different semiphenomenological approaches are developed ranging from microscopic-like models and Landau theories to membrane models. At present, the applications of computer simulations for treating such simple self-assembly effects as the spontaneous appearance of micelles above the critical micelle concentration (CMC) of a surfactant in water are possible only for very simple systems. There are a few problems with this treatment, such as a sufficiently small value of the CMC and the large number of particles that are needed for the simulations, the problem of long times of performing simulation for obtaining self-assembling aggregates, etc.

Peter Monson: In some sense the development of simulation has had a negative consequence. With death of John Barker, studies such as his on intermolecular forces have shut down and perhaps his pioneering work might be lost.

Douglas Henderson: I agree with Peter and share his admiration for John Barker. In spirit of whimsey, I wonder if simulations should be restricted to those who had first served an apprenticeship in analytical theory.

\section{Instead of conclusions}

Ariel Chialvo: The most typical scenario in the study of soft matter is the one where we have to contend with two realms [worlds], i.e., the real and the mathematical worlds, as well as three interconnected targets, namely: laboratory experiments, theoretical developments, and molecular-based simulations. In the quest for understanding of soft matter behaviour, neither one of these targets can stand alone, but integrated with one another as indicated in figure 1.

The interconnection hinges around three essential ingredients associated with this concerted effort are as follows:

(i) the realism of a [mathematical] model in describing the real world,

(ii) the accuracy of the theory in representing the model, and

(iii) the validity [adequacy] of the molecular-based simulation in representing the model.

Thus, we must always address all three items to get the most from the interplay between theory, simulation, and experiment. Otherwise, we may find ourselves confronted with the dilemma about whether any disagreement in the comparison either 


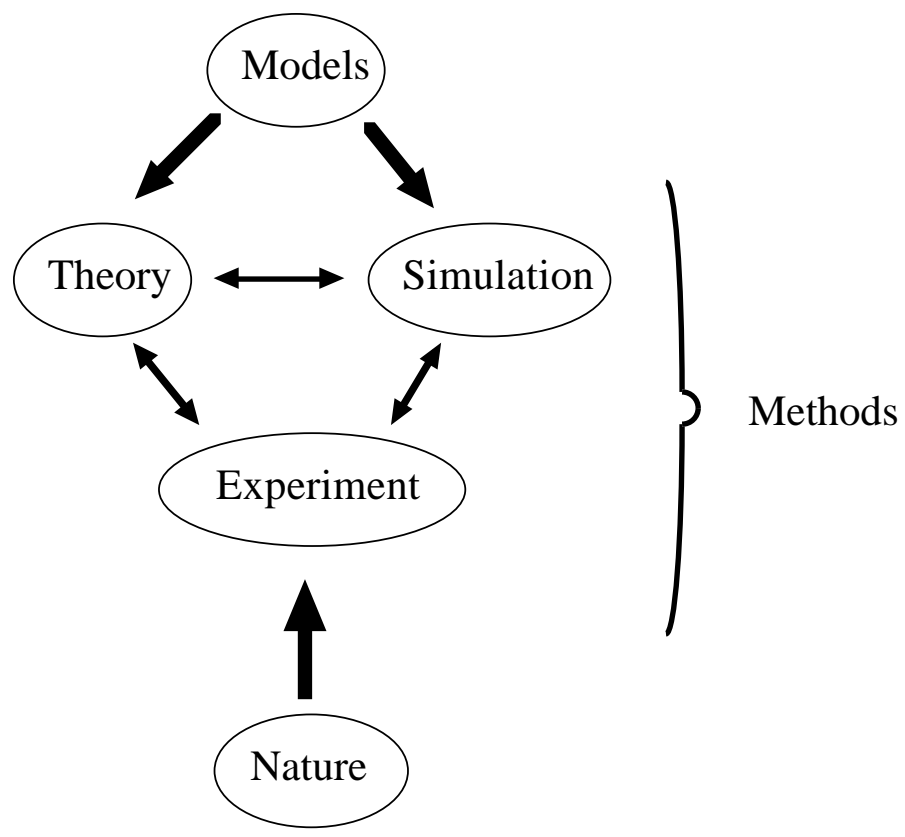

Figure 1. Schematic representation of the targeted systems and the tools of the trade used in their study

(i) between theory and experiment results from an unrealistic [potential] model, or (ii) between simulation and theory results from inaccuracies in the theory. To solve this dilemma we should first establish the validity of the molecular-based simulation methodology independently from the associated theory or the experiment. Consequently, we can inquire about the realism of a particular mathematical [intermolecular potentia] model by comparing the simulation results for that model with the corresponding experimental data. At the same time, we can compare these simulation results with those generated by the theoretical counterpart involving precisely the same potential model, and thus, we can assess the accuracy of the theory in representing the mathematical model. In summary, and as my simulation mentor used to say: 'molecular simulation is a tool whose value strongly depends on the skills of the practitioner who operates it'. Therefore, 'we should never forget that the realm we create is an artificial world, to mimic the real world which we hope to understand, eventually'.

\section{References}

1. Allen M.P. and Tildesley D.J. Computer Simulations of Liquids. Oxford, Clarendon, 1987.

2. Frenkel D. and Smit B. Understanding Molecular Simulation. New-York, Academic, 1996. 


\title{
Матеріали круглого столу "Аналітичні методи та комп'ютерний експеримент в теоріі м'якої речовини"
}

\section{Зібрано і відредаговано}

\author{
А.Трохимчуком ${ }^{1}$, Д.Гендерсоном ${ }^{2}$, Л.Лі ${ }^{3}$
}

1 Інститут фізики конденсованих систем НАН України, 79011 Львів, вул. Свєнціцького, 1

2 Факультет хімії та біохімії, Університет Брайхем Янг, Прово, UT 84602, США

3 Школа хімічної інженерії та матеріалознавства, Університет штату Оклахоми, Норман, ОК 73019, США

У статті подано матеріали дискусії щодо розвитку, сучасного стану та перспектив аналітичних методів і комп'ютерного моделювання в теоріі м'якої речовини, що відбулася підчас Наради із сучасних проблем теорії м'якої речовини (27-31 серпня 2000 р., м. Львів).

Ключові слова: аналітична теорія, комп'ютерне моделювання

PACS: $07.05 . T$ 